PROCEEDINGS OF THE

AMERICAN MATHEMATICAL SOCIETY

Volume 133, Number 10, Pages 3079-3085

S 0002-9939(05)08077-9

Article electronically published on April 20, 2005

\title{
A NOTE ON THE ZERO MACH NUMBER LIMIT OF COMPRESSIBLE EULER EQUATIONS
}

\author{
WEN-AN YONG
}

(Communicated by M. Gregory Forest)

\begin{abstract}
This note presents a short and elementary justification of the classical zero Mach number limit for isentropic compressible Euler equations with prepared initial data. We also show the existence of smooth compressible flows, with the Mach number sufficiently small, on the (finite) time interval where the incompressible Euler equations have smooth solutions.
\end{abstract}

\section{INTRODUCTION}

In a suitable nondimensional form (see, e.g., [5]), the compressible Euler equations for an isentropic fluid read as

$$
\begin{aligned}
\rho_{t}+\operatorname{div}(\rho v) & =0 \\
(\rho v)_{t}+\operatorname{div}(\rho v \otimes v)+\epsilon^{-2} \nabla p & =0 .
\end{aligned}
$$

Here $\rho=\rho(x, t)$ is the density function of $(x, t) \in \Omega \times[0, \infty)$ with $\Omega \subset \mathbb{R}^{d}$, $v=v(x, t)=\left(v_{1}, v_{2}, \cdots, v_{d}\right)(x, t)$ is the fluid velocity, $\epsilon$ is the Mach number, and the pressure $p=p(\rho)$ is a given strictly increasing smooth function of $\rho>0$. Throughout this paper, $\Omega$ is assumed to be $\mathbb{R}^{d}$ or the $d$-dimensional torus and (1.1) is supplemented with initial data

$$
(\rho, v)(x, 0)=(\bar{\rho}(x, \epsilon), \bar{v}(x, \epsilon)) .
$$

The interest is to investigate the limit when $\epsilon$ goes to zero. This limit problem was first studied in [3, 4, 5] and has attracted much attention since then. The interested reader is referred to [6] for a comprehensive survey of the literature.

In this note, we present a short and elementary approach to the above limit problem. This approach is based on the convergence-stability lemma (Lemma 9.1) in 9] - a continuation principle first formulated in 9] for general (hyperbolic) singular limt problems.

Our result can be roughly stated as follows. Suppose the initial data in (1.2) are smooth and have the form

$$
\bar{\rho}(x, \epsilon)=\rho_{0}+O\left(\epsilon^{2}\right), \quad \bar{v}(x, \epsilon)=\bar{v}(x, 0)+O(\epsilon)
$$

Received by the editors June 4, 2004.

2000 Mathematics Subject Classification. Primary 35B25, 35L45, 76N10.

Key words and phrases. Compressible Euler equations, incompressible limit, symmetrizable hyperbolic systems, continuation principle, energy estimates.

(C)2005 American Mathematical Society Reverts to public domain 28 years from publication 
with $\rho_{0}>0$ a constant and $\bar{v}(x, 0)$ solenoidal. Let $\left[0, T_{*}\right]$ be a (finite) time interval where the incompressible Euler equations

$$
\begin{aligned}
\operatorname{div} w & =0, \\
\rho_{0}\left(w_{t}+w \cdot \nabla w\right)+\nabla \pi & =0
\end{aligned}
$$

with $w(x, 0)=\bar{v}(x, 0)$ have a smooth solution $(\pi, w)$. Then, for $\epsilon$ sufficiently small, the compressible Euler equations (1.1) with (1.2) have a unique smooth solution $\left(\rho^{\epsilon}, v^{\epsilon}\right)$ defined for $(x, t) \in \Omega \times\left[0, T_{*}\right]$ and satisfying

$$
\rho^{\epsilon}=\rho_{0}+O\left(\epsilon^{2}\right), \quad v^{\epsilon}=w+O(\epsilon) .
$$

For details and comparison to existing results, see the next section. For the existence of the smooth solution $(\pi, w)$, see [2, 7].

Note that our analysis can be extended to the same limit problem of the NavierStokes equations and requires $T_{*}$ to be finite. However, for the Navier-Stokes equations of heat-conducting fluid flows, it is possible to study the Mach number limit for $T_{*}=\infty[1]$.

Notation. $|U|$ denotes some norm of a vector or matrix $U$. For a nonnegative integer $k, H^{k}=H^{k}(\Omega)$ denotes the usual $L^{2}$-type Sobolev space of order $k$. We write $\|\cdot\|_{k}$ for the standard norm of $H^{k}$ and $\|\cdot\|$ for $\|\cdot\|_{0}$. When $U$ is a function of another variable $t$ as well as $x$, we write $\|U(\cdot, t)\|_{k}$ to recall that the norm is taken with respect to $x$ while $t$ is viewed as a parameter. In addition, we denote by $C([0, T], \mathbf{X})$ (resp. $\left.C^{1}([0, T], \mathbf{X})\right)$ the space of continuous (resp. continuously differentiable) functions on $[0, T]$ with values in a Banach space $\mathbf{X}$.

\section{RESULTS}

First of all, we rewrite the compressible Euler equations (1.1) as a symmetrizable hyperbolic system. Since $p=p(\rho)$ is strictly increasing, it has an inverse $\rho=\rho(p)$.

Set $q(p)=\left[\rho(p) p^{\prime}(\rho(p))\right]^{-1}$. Then the Euler equations (1.1) for smooth solutions can be rewritten as

$$
\begin{aligned}
q(p)\left(p_{t}+v \cdot \nabla p\right)+\operatorname{div} v & =0, \\
\rho(p)\left(v_{t}+v \cdot \nabla v\right)+\epsilon^{-2} \nabla p & =0 .
\end{aligned}
$$

Following [5], we introduce

$$
\tilde{p}=\left(p-p_{0}\right) / \epsilon \quad \text { and } \quad \tilde{v}=v
$$

with $p_{0}=p\left(\rho_{0}\right)>0$. Then (2.1) can be rewritten as

$$
\begin{aligned}
q\left(p_{0}+\epsilon \tilde{p}\right)\left(\tilde{p}_{t}+\tilde{v} \cdot \nabla \tilde{p}\right)+\epsilon^{-1} \operatorname{div} \tilde{v} & =0, \\
\rho\left(p_{0}+\epsilon \tilde{p}\right)\left(\tilde{v}_{t}+\tilde{v} \cdot \nabla \tilde{v}\right)+\epsilon^{-1} \nabla \tilde{p} & =0 .
\end{aligned}
$$

In the vector form, we arrive at, for $U=(\tilde{p}, \tilde{v})$,

$$
A_{0}(\epsilon \tilde{p}) U_{t}+\sum_{j=1}^{d}\left(\tilde{v}_{j} A_{0}(\epsilon \tilde{p})+\epsilon^{-1} C_{j}\right) U_{x_{j}}=0 .
$$

Here $A_{0}=A_{0}(\epsilon \tilde{p})$ is diagonal and positive definite, and $C_{j}$ is constant and symmetric. 
For $\epsilon \in(0,1]$, the state space for (2.3) is obviously $G=\left(-p_{0},+\infty\right) \times \mathbb{R}^{d}$. Assume the initial data $\bar{U}=(\bar{p}(x, \epsilon), \bar{v}(x, \epsilon))$ for (2.3) satisfies

$$
\{\bar{U}(x, \epsilon):(x, \epsilon) \in \Omega \times(0,1]\} \in\left[-p_{0} / 2, C\right] \times[-C, C]^{d} \equiv G_{0} \subset \subset G
$$

with $C>0$ a constant and $\bar{U}(\cdot, \epsilon) \in H^{s}$ with $s>d / 2+1$ an integer. Fix $\epsilon$. According to the local existence theory for IVPs of symmetrizable hyperbolic systems (see Theorem 2.1 in [5]), for each convex open subset $G_{1}$ satisfying $G_{0} \subset \subset G_{1} \subset \subset G$, there exists $T>0$ so that (2.3) has a unique classical solution

$$
U^{\epsilon} \in C\left([0, T], H^{s}\right) \quad \text { and } \quad U^{\epsilon}(x, t) \in G_{1} \quad \forall(x, t) \in \Omega \times[0, T] .
$$

Define

(2.4) $T_{\epsilon}=\sup \left\{T>0: U^{\epsilon} \in C\left([0, T], H^{s}\right), \quad U^{\epsilon}(x, t) \in G_{1} \quad \forall(x, t) \in \Omega \times[0, T]\right\}$.

Namely, $\left[0, T_{\epsilon}\right)$ is the maximal time interval of $H^{s}$ existence. Note that $T_{\epsilon}$ depends on $G_{1}$ and may tend to zero as $\epsilon$ goes to 0 .

To show that $\underline{\lim }_{\epsilon \rightarrow 0} T_{\epsilon}>0$, we will prove the following theorem in the next section.

Theorem 2.1. Suppose the initial data $\bar{U}(x, \epsilon)$ satisfy $\|\bar{p}(\cdot, \epsilon), \bar{v}(\cdot, \epsilon)-\bar{v}(\cdot, 0)\|_{s}=$ $O(\epsilon)$ and $\operatorname{div} \bar{v}(\cdot, 0)=0$. Let $(\pi, w)$ be a smooth solution to the incompressible Euler equations (1.3) with $w(x, 0)=\bar{v}(x, 0)$. If

$$
(\pi, w) \in C\left(\left[0, T_{*}\right], H^{s+1}\right) \cap C^{1}\left(\left[0, T_{*}\right], H^{s}\right)
$$

with $T_{*}>0$ finite, then there are positive constants $\epsilon_{0}$ and $K$ such that, for $\epsilon \leq \epsilon_{0}$,

$$
\left\|U^{\epsilon}(\cdot, t)-(\epsilon \pi, w)(\cdot, t)\right\|_{s} \leq K \epsilon
$$

for $t \in\left[0, \min \left\{T_{*}, T_{\epsilon}\right\}\right)$.

Having this theorem, we slightly modify the argument in [8] to prove

Theorem 2.2. Under the conditions of Theorem 2.1 for any $G_{1}$ satisfying

$$
G_{0} \bigcup_{x, t, \epsilon}\{(\epsilon \pi, w)(x, t)\} \subset \subset G_{1} \subset \subset G,
$$

$T_{\epsilon}\left(G_{1}\right)>T_{*}$ holds for $\epsilon>0$ sufficiently small.

Proof. Otherwise, there is a $G_{1}$ satisfying (2.5) and a sequence $\left\{\epsilon_{k}\right\}_{k \geq 1}$ such that $\lim _{k \rightarrow \infty} \epsilon_{k}=0$ and $T_{\epsilon_{k}}=T_{\epsilon_{k}}\left(G_{1}\right) \leq T_{*}$. Then there exists $\tilde{G}$ satisfying

$$
\bigcup_{x, t, \epsilon}\{(\epsilon \pi, w)(x, t)\} \subset \subset \tilde{G} \subset \subset G_{1} .
$$

Moreover, we deduce from Sobolev's embedding theorem and Theorem 2.1 that

$$
\left|U^{\epsilon}(x, t)-(\epsilon \pi, w)(x, t)\right| \leq \text { const. }\left\|U^{\epsilon}(\cdot, t)-(\epsilon \pi, w)(\cdot, t)\right\|_{s} \leq \text { const. } K \epsilon .
$$

Thus, there is a $k$ such that $U^{\epsilon_{k}}(x, t) \in \tilde{G}$ for all $(x, t) \in \Omega \times\left[0, T_{\epsilon_{k}}\right)$. On the other hand, it follows from

$$
\left\|U^{\epsilon}(\cdot, t)\right\|_{s} \leq\left\|U^{\epsilon}(\cdot, t)-(\epsilon \pi, w)(\cdot, t)\right\|_{s}+\|(\epsilon \pi, w)(\cdot, t)\|_{s} \leq K \epsilon_{0}+\|(\epsilon \pi, w)(\cdot, t)\|_{s}
$$

that $\left\|U^{\epsilon_{k}}(\cdot, t)\right\|_{s}$ is bounded uniformly with respect to $t \in\left[0, T_{\epsilon_{k}}\right)$. Now we could apply Theorem 2.1 in [5], beginning at a time $t$ less than $T_{\epsilon_{k}}$ ( $k$ is fixed here!), to continue the solution beyond $T_{\epsilon_{k}}\left(G_{1}\right)$. This contradicts the definition of $T_{\epsilon_{k}}\left(G_{1}\right)$ in (2.4) and, hence, the proof is complete. 
We make two remarks about the above theorems.

Remark 2.1. In case $(\pi, w)$ is defined globally in time and the conditions of Theorem 2.1 hold for $T_{*}=\infty$, we actually prove the following existence result for (2.3): For any $T<\infty$, there is a neigborhood of $\epsilon=0$ such that for all $\epsilon$ in the neighborhood, (2.3) with initial data $\bar{U}(x, \epsilon)$ has a unique classical solution

$$
U^{\epsilon} \in C\left([0, T], H^{s}\right) .
$$

Moreover, the error estimate in Theorem 2.1 holds, for $t \leq T$, with $K$ depending on $T$.

Remark 2.2. In comparison with previous works [3, 4, 5] 6], especially [4, 5], our approximate solution $(\epsilon \pi, w)$ is the simplest. Thus, the regularity requirement on the initial data and approximate solution is the least. Moreover, the establishment of Theorem 2.2 simplifies the analysis considerably.

Finally, we mention that Theorem 2.2 is a special case of the convergence-stability lemma (Lemma 9.1) in [9] - a continuation principle formulated in [9] for general (hyperbolic) singular limit problems.

\section{A proof of Theorem 2.1}

This section is devoted to proving Theorem 2.1. Note that

$$
p_{\epsilon}=\epsilon \pi \quad \text { and } \quad v_{\epsilon}=w
$$

satisfy

$$
\begin{aligned}
q\left(p_{0}+\epsilon p_{\epsilon}\right)\left(p_{\epsilon t}+v_{\epsilon} \cdot \nabla p_{\epsilon}\right)+\epsilon^{-1} \operatorname{div} v_{\epsilon} & =\epsilon q\left(p_{0}+\epsilon^{2} \pi\right)\left(\pi_{t}+w \cdot \nabla \pi\right), \\
\rho\left(p_{0}+\epsilon p_{\epsilon}\right)\left(v_{\epsilon t}+v_{\epsilon} \cdot \nabla v_{\epsilon}\right)+\epsilon^{-1} \nabla p_{\epsilon} & =\left[\rho\left(p_{0}+\epsilon^{2} \pi\right)-\rho\left(p_{0}\right)\right]\left(w_{t}+w \cdot \nabla w\right) .
\end{aligned}
$$

Namely, $U_{\epsilon}=(\epsilon \pi, w)$ satisfies

$$
U_{\epsilon t}+\sum_{j=1}^{d} A_{j}\left(U_{\epsilon}, \epsilon\right) U_{\epsilon x_{j}}=\left(\left(1-\frac{\rho\left(p_{0}\right)}{\rho\left(p_{0}+\epsilon^{2} \pi\right)}\right)\left(w_{t}+w \cdot \nabla w\right)\right) \equiv R,
$$

where $A_{j}(U, \epsilon)=v_{j} I_{d+1}+\epsilon^{-1} A_{0}(\epsilon p)^{-1} C_{j}$ with $I_{d+1}$ the unit matrix of order $(d+1)$. Since $(\pi, w) \in C\left(\left[0, T_{*}\right], H^{s+1}\right) \cap C^{1}\left(\left[0, T_{*}\right], H^{s}\right)$ is assumed in Theorem 2.1, we have

$$
\max _{t \in\left[0, T_{*}\right]}\|R(\cdot, t)\|_{s} \leq C \epsilon .
$$

Here and below, $C$ denotes a generic constant that can change from line to line.

From (2.3) and (3.1) we compute that $E=U_{\epsilon}-U^{\epsilon}$ satisfies

$$
E_{t}+\sum_{j} A_{j}\left(U^{\epsilon}, \epsilon\right) E_{x_{j}}=R+\sum_{j}\left[A_{j}\left(U^{\epsilon}, \epsilon\right)-A_{j}\left(U_{\epsilon}, \epsilon\right)\right] U_{\epsilon x_{j}} .
$$

Differentiating this equation with $\partial^{\alpha}$ for any multi-index $\alpha$ satisfying $|\alpha| \leq s$ and setting $E_{\alpha}=\partial^{\alpha} E$, we get

$$
E_{\alpha t}+\sum_{j} A_{j}\left(U^{\epsilon}, \epsilon\right) E_{\alpha x_{j}}=R_{\alpha}+F^{\alpha}
$$

with

$$
F^{\alpha}=\left\{\sum_{j}\left[A_{j}\left(U^{\epsilon}, \epsilon\right)-A_{j}\left(U_{\epsilon}, \epsilon\right)\right] U_{\epsilon x_{j}}\right\}_{\alpha}+\sum_{j}\left\{A_{j}\left(U^{\epsilon}, \epsilon\right) E_{\alpha x_{j}}-\left[A_{j}\left(U^{\epsilon}, \epsilon\right) E_{x_{j}}\right]_{\alpha}\right\} .
$$


Recall that $A_{0}^{\epsilon} \equiv A_{0}\left(h_{0}+\epsilon h^{\epsilon}\right)$ and $A_{0}^{\epsilon} A_{j}\left(U^{\epsilon}, \epsilon\right)(j=1,2, \cdots, d)$ are all symmetric. Let $E_{\alpha}^{T}$ be the transpose of $E_{\alpha}$. Multiplying (3.3) with $E_{\alpha}^{T} A_{0}^{\epsilon}$ from the left and setting $e\left(E_{\alpha}\right)=E_{\alpha}^{T} A_{0}^{\epsilon} E_{\alpha}$, we get

$$
\begin{gathered}
e\left(E_{\alpha}\right)_{t}+\sum_{j}\left\{E_{\alpha}^{T} A_{0}^{\epsilon} A_{j}\left(U^{\epsilon}, \epsilon\right) E_{\alpha}\right\}_{x_{j}}=2 \operatorname{Re} E_{\alpha}^{T} A_{0}^{\epsilon}\left(R_{\alpha}+F^{\alpha}\right) \\
+E_{\alpha}^{T}\left\{\frac{\partial A_{0}^{\epsilon}}{\partial t}+\sum_{j} \frac{\partial\left[A_{0}^{\epsilon} A_{j}\left(U^{\epsilon}, \epsilon\right)\right]}{\partial x_{j}}\right\} E_{\alpha} .
\end{gathered}
$$

Now we estimate various terms in (3.4). Note that our estimates only need to be done for $t \in\left[0, \min \left\{T_{*}, T_{\epsilon}\right\}\right.$ ), in which both $U^{\epsilon}$ and $U_{\epsilon}$ are regular enough and take values in a convex compact subset of the state space. In particular, we have

$$
\begin{aligned}
C^{-1}\left|E_{\alpha}\right|^{2} & \leq e\left(E_{\alpha}\right) \leq C\left|E_{\alpha}\right|^{2}, \\
2 \operatorname{Re} E_{\alpha}^{T} A_{0}^{\epsilon}\left(R_{\alpha}+F^{\alpha}\right) & \leq C\left|E_{\alpha}\right|^{2}+C\left|R_{\alpha}\right|^{2}+C\left|F^{\alpha}\right|^{2} .
\end{aligned}
$$

Moreover, since $U^{\epsilon}$ is an exact solution to (2.3) or (2.2), it is not difficult to get

$\frac{\partial A_{0}^{\epsilon}}{\partial t}+\sum_{j} \frac{\partial\left[A_{0}^{\epsilon} A_{j}\left(U^{\epsilon}, \epsilon\right)\right]}{\partial x_{j}}=A_{0}^{\epsilon} \operatorname{div} v^{\epsilon}+A_{0 t}^{\epsilon}+v^{\epsilon} \cdot \nabla A_{0}^{\epsilon}=\left(A_{0}^{\epsilon}-\frac{A_{0}^{\prime}\left(\epsilon h^{\epsilon}\right)}{q\left(h_{0}+\epsilon h^{\epsilon}\right)}\right) \operatorname{div} v^{\epsilon}$.

Because $s>d / 2+1$, we use Sobolev's embedding theorem to obtain

$\frac{\partial A_{0}^{\epsilon}}{\partial t}+\sum_{j} \frac{\partial\left[A_{0}^{\epsilon} A_{j}\left(U^{\epsilon}, \epsilon\right)\right]}{\partial x_{j}} \leq C\left|\operatorname{div} v^{\epsilon}\right| \leq C\left|\operatorname{div} v_{\epsilon}\right|+C\left|\operatorname{div}\left(v_{\epsilon}-v^{\epsilon}\right)\right| \leq C+C\|E\|_{s}$.

Next we estimate $\left\|F^{\alpha}\right\|$ with the help of the Moser-type calculus inequalities in Sobolev spaces [5]. For the first term, we use the relation

$$
\begin{aligned}
& A_{j}\left(U^{\epsilon}, \epsilon\right)-A_{j}\left(U_{\epsilon}, \epsilon\right)=\left(v_{j}^{\epsilon}-v_{j \epsilon}\right) I_{d+1}+\epsilon^{-1}\left[\left(A_{0}^{\epsilon}\right)^{-1}-A_{0}\left(\epsilon p_{\epsilon}\right)^{-1}\right] C_{j} \\
= & \left(v_{j}^{\epsilon}-v_{j \epsilon}\right) I_{d+1}-\left(p^{\epsilon}-p_{\epsilon}\right)\left(A_{0}^{-2} A_{0}^{\prime}\right)\left(\epsilon p_{\epsilon}+\epsilon \theta\left(p^{\epsilon}-p_{\epsilon}\right)\right) C_{j},
\end{aligned}
$$

with $\theta \in[0,1]$, and the boundedness of $\|(\epsilon \pi, w)(\cdot, t)\|_{s+1}$ to conclude that

$$
\begin{aligned}
\left\|\left\{\left[A_{j}\left(U^{\epsilon}, \epsilon\right)-A_{j}\left(U_{\epsilon}, \epsilon\right)\right] U_{\epsilon x_{j}}\right\}_{\alpha}\right\| & \leq C\left\|U_{\epsilon x_{j}}\right\|_{s}\left\|A_{j}\left(U^{\epsilon}, \epsilon\right)-A_{j}\left(U_{\epsilon}, \epsilon\right)\right\|_{|\alpha|} \\
& \leq C\left(1+\left\|p_{\epsilon}+\theta\left(p^{\epsilon}-p_{\epsilon}\right)\right\|_{s}^{s}\right)\|E\|_{|\alpha|} \\
& \leq C\left(1+\|E\|_{s}^{s}\right)\|E\|_{|\alpha|} .
\end{aligned}
$$

For the second term, since

$$
\begin{aligned}
& A_{j}\left(U^{\epsilon}, \epsilon\right) E_{\alpha x_{j}}-\left[A_{j}\left(U^{\epsilon}, \epsilon\right) E_{x_{j}}\right]_{\alpha}=-\sum_{0<\beta \leq \alpha}\left(\begin{array}{c}
\alpha \\
\beta
\end{array}\right) \partial^{\beta} A_{j}\left(U^{\epsilon}, \epsilon\right) \partial^{\alpha-\beta} E_{x_{j}} \\
= & -\sum_{0<\beta \leq \alpha}\left(\begin{array}{c}
\alpha \\
\beta
\end{array}\right) \partial^{\beta}\left[v_{j}^{\epsilon} I_{d+1}+\epsilon^{-1}\left(A_{0}^{\epsilon}\right)^{-1} C_{j}\right] \partial^{\alpha-\beta} E_{x_{j}},
\end{aligned}
$$

we have

$$
\begin{aligned}
\left\|A_{j}\left(U^{\epsilon}, \epsilon\right) E_{\alpha x_{j}}-\left[A_{j}\left(U^{\epsilon}, \epsilon\right) E_{x_{j}}\right]_{\alpha}\right\| & \leq C\left(\|E\|_{s}+\left\|p^{\epsilon}\right\|_{s}^{s}\right)\left\|E_{x_{j}}\right\|_{|\alpha|-1} \\
& \leq C\left(1+\|E\|_{s}^{s}\right)\|E\|_{|\alpha|} .
\end{aligned}
$$

Putting the above two estimates together, we get

$$
\left\|F^{\alpha}\right\| \leq C\left(1+\|E\|_{s}^{s}\right)\|E\|_{|\alpha|} .
$$


Substituting (3.6)-(3.8) into (3.4) and integrating it over $x \in \Omega$ yields

$$
\frac{d}{d t} \int_{\Omega} e\left(E_{\alpha}\right) d x \leq C\left\|R_{\alpha}\right\|^{2}+C\left(1+\|E\|_{s}^{2 s}\right)\|E\|_{|\alpha|}^{2} .
$$

With (3.5), we integrate (3.9) from 0 to $T$ with $T<\min \left\{T_{\epsilon}, T_{*}\right\}$ to obtain

$$
\left\|E_{\alpha}(T)\right\|^{2} \leq C\left\|E_{\alpha}(0)\right\|^{2}+C \int_{0}^{T}\left\|R_{\alpha}(t)\right\|^{2} d t+C \int_{0}^{T}\left(1+\|E(t)\|_{s}^{2 s}\right)\|E(t)\|_{|\alpha|}^{2} d t .
$$

Summing up this inequality for all $\alpha$ with $|\alpha| \leq s$, we get

$$
\|E(T)\|_{s}^{2} \leq C\|E(0)\|_{s}^{2}+C \int_{0}^{T_{*}}\|R(t)\|_{s}^{2} d t+C \int_{0}^{T}\left(1+\|E(t)\|_{s}^{2 s}\right)\|E(t)\|_{s}^{2} d t .
$$

Since $\|E(0)\|_{s}^{2}+\int_{0}^{T_{*}}\|R(t)\|_{s}^{2} d t=O\left(\epsilon^{2}\right)$, we apply Gronwall's lemma to (3.10) to get

$$
\|E(T)\|_{s}^{2} \leq C \epsilon^{2} \exp \left[C \int_{0}^{T}\left(1+\|E(t)\|_{s}^{2 s}\right) d t\right] \equiv \Phi(T) .
$$

Thus, we have

$$
\Phi^{\prime}(t)=C\left(1+\|E(t)\|_{s}^{2 s}\right) \Phi(t) \leq C \Phi(t)+C \Phi^{s+1}(t) .
$$

Applying the nonlinear Gronwall-type inequality in [8] to this inequality yields

$$
\|E(t)\|_{s}^{2} \leq \Phi(t) \leq \exp \left(C T_{*}\right)
$$

for all $t \in\left[0, \min \left\{T_{\epsilon}, T_{*}\right\}\right)$ if $\Phi(0)=C \epsilon^{2}<\exp \left(-C T_{*}\right)$. Because of (B.11), there exists a constant $K$, independent of $\epsilon$, such that

$$
\|E(t)\|_{s} \leq K \epsilon
$$

for all $t \in\left[0, \min \left\{T_{\epsilon}, T_{*}\right\}\right)$. This completes the proof.

\section{ACKNOWLEDGEMENTS}

The author thanks the anonymous referees for valuable suggestions. This work was supported by the Deutsche Forschungsgemeinschaft through SFB 359 at the University of Heidelberg and by the European TMR-Network "Hyperbolic and Kinetic Equations".

\section{REFERENCES}

[1] T. Hagstrom and J. Lorenz, On the stability of approximate solutions of hyperbolic-parabolic systems and the all-time existence of smooth, slightly compressible flows, Indiana Univ. Math. J. 51 (2002), pp. 1339-1387. MR1948453 (2004h:35188)

[2] T. Kato, Nonstationary flows of viscous and ideal fluids in $R^{3}$, J. Funct. Anal. 9 (1972), pp. 296-305. MR0481652 (58:1753)

[3] S. Klainerman and A. Majda, Singular limits of quasilinear hyperbolic systems with large parameters and the incompressible limit of compressible fluids, Commun. Pure Appl. Math. 34 (1981), pp. 481-524. MR0615627 (84d:35089)

[4] S. Klainerman and A. Majda, Compressible and incompressible fluids, Commun. Pure Appl. Math. 35 (1982), pp. 629-651. MR0668409 (84a:35264)

[5] A. Majda, Compressible fluid flow and systems of conservation laws in several space variables, Springer, New York, 1984. MR.0748308 (85e:35077)

[6] G. Métivier and S. Schochet, The incompressible limit of the non-isentropic Euler equations, Arch. Rational Mech. Anal. 158 (2001), pp. 61-90. MR.1834114 (2002d:76095) 
[7] R. Teman, Local existence of solutions of the Euler equations of incompressible perfect fluids, in Turbulence and the Navier-Stokes equations, Springer, New York, 1976, pp. 184-194. MR:0467033(57:6902)

[8] W.-A. Yong, Singular perturbations of first-order hyperbolic systems with stiff source terms, J. Differ. Equations 155 (1999), pp. 89-132. MR1693210 (2000c:35011)

[9] W.-A. Yong, Basic aspects of hyperbolic relaxation systems, in Advances in the Theory of Shock Waves, Birkhäuser, Boston, 2001, pp. 259-305. MR1842777 (2002g:35136)

IWR, Universität Heidelberg, Im Neuenheimer Feld 294, 69120 Heidelberg, Germany

E-mail address: yong.wen-an@iwr.uni-heidelberg.de 